\title{
Hyperoxia disrupts pulmonary epithelial barrier in newborn rats via the deterioration of occludin and ZO-1
}

Kai You', Xuewen $X u^{2}$, Jianhua $F u^{1}$, Shuyan $X u^{1}$, Xiaohong Yue ${ }^{3}$, Zhiling $Y u^{1}$ and Xindong Xue ${ }^{1 *}$

\begin{abstract}
Background: Prolonged exposure to hyperoxia in neonates can cause hyperoxic acute lung injury (HALI), which is characterized by increased pulmonary permeability and diffuse infiltration of various inflammatory cells. Disruption of the epithelial barrier may lead to altered pulmonary permeability and maintenance of barrier properties requires intact epithelial tight junctions (TJs). However, in neonatal animals, relatively little is known about how the TJ proteins are expressed in the pulmonary epithelium, including whether expression of TJ proteins is regulated in response to hyperoxia exposure. This study determines whether changes in tight junctions play an important role in disruption of the pulmonary epithelial barrier during hyperoxic acute lung injury.

Methods: Newborn rats, randomly divided into two groups, were exposed to hyperoxia (95\% oxygen) or normoxia for 1-7 days, and the severity of lung injury was assessed; location and expression of key tight junction protein occludin and ZO-1 were examined by immunofluorescence staining and immunobloting; messenger RNA in lung tissue was studied by RT-PCR; transmission electron microscopy study was performed for the detection of tight junction morphology.

Results: We found that different durations of hyperoxia exposure caused different degrees of lung injury in newborn rats. Treatment with hyperoxia for prolonged duration contributed to more serious lung injury, which was characterized by increased wet-to-dry ratio, extravascular lung water content, and bronchoalveolar lavage fluid (BALF):serum FD4 ratio. Transmission electron microscopy study demonstrated that hyperoxia destroyed the structure of tight junctions and prolonged hyperoxia exposure, enhancing the structure destruction. The results were compatible with pathohistologic findings. We found that hyperoxia markedly disrupted the membrane localization and downregulated the cytoplasm expression of the key tight junction proteins occludin and ZO-1 in the alveolar epithelium by immunofluorescence. The changes of messenger RNA and protein expression of occludin and ZO-1 in lung tissue detected by RT-PCR and immunoblotting were consistent with the degree of lung injury.

Conclusions: These data suggest that the disruption of the pulmonary epithelial barrier induced by hyperoxia is, at least in part, due to massive deterioration in the expression and localization of key TJ proteins.
\end{abstract}

Keywords: Acute lung injury, Hyperoxia, Newborn, Permeability, Tight Junction

\footnotetext{
* Correspondence: xuexd@sj-hospital.org

'Department of Pediatrics, Shengjing Hospital of China Medical University,

Shenyang 110004, China

Full list of author information is available at the end of the article
} 


\section{Background}

Therapy with a high concentration of oxygen (hyperoxia) is often necessary to treat neonates with respiratory failure in neonatal intensive care units. However, one of the main complications is hyperoxia-induced lung injury, which may later result in serious sequela, such as bronchopulmonary dysplasia (BPD) [1,2]. The neonates with BPD continue to show decreased lung capacity at school age and even as young adolescents [3-5]. BPD has a negative impact not only on short-term and long-term pulmonary function but also on overall growth and neurodevelopment of these infants [6,7]. However, the mechanism of hyperoxia-induced lung injury is still not completely understood and currently there are no evidence-based effective strategies to prevent or treat this disease. Thus, there is an urgent need to understand how hyperoxia exposure initially influences pulmonary structure and function and how this lastingly disrupts lung development.

It is well known that the major pathologic finding associated with hyperoxia exposure at the early stage is hyperoxia-induced acute lung injury, which is frequently accompanied by an inflammatory response within lungs $[8,9]$. This response is characterized by increased pulmonary permeability and diffuse infiltration of various inflammatory cells [10-12]. Disruption of the epithelial barrier may lead to altered pulmonary permeability and airways fluid accumulation [13,14]. However, little attention has been done on the molecular mechanisms involved in disruption of the pulmonary epithelial barrier in neonatal hyperoxic lung injury.

The permeability barrier in terminal airspaces of the lung is due in large part to tight junctions between alveolar epithelial cells, which are continuous, circumferential, belt-like structures and regulate the flow of molecules between apical and basolateral compartments $[15,16]$. Tight junction is a primary barrier that regulates the diffusion of solutes through the paracellular pathway. Occludin and zonula occludens 1 (ZO-1) are two well characterized proteins in tight junctions. Occludin is a transmembrane protein which provides most of the barrier function of the tight junction $[17,18]$. Zonula occludens 1 is an intracellular protein, located between occludin and cytoskeletal proteins and thought to affect paracellular permeability [19]. Occludin and ZO-1 proteins form the intact intercellular barrier at tight junctions, and differences in the expression of these key TJ proteins account for differences in barrier function and paracellular permeability among epithelia [20-23]. For example, previous studies in intestinal epithelia have shown that serious impairment of intestinal barrier function is associated with an increased permeability and morphological changes through downregulation and localization shift of occluding [24,25]. Others have reported that changes in occludin and $\mathrm{ZO}-1$ expression in response to environmental stimuli may account for changes in barrier properties in the pulmonary epithelia [24,26-28].

However, our current understanding of the structural components and regulation of tight junctions in the alveolar epithelium is insufficient, and previous studies were mainly performed in adult animals or cells and have scarcely been examined in the neonatal period. The primary objective of this study was to determine whether there were changes in alveolar barrier structure and function in newborn rats exposed to hyperoxia and whether tight junction proteins were differentially expressed in the lung during experimental acute lung injury induced by hyperoxia and whether these changes account for impairment in alveolar barrier properties and are relevant to pulmonary edema formation.

\section{Methods}

\section{Animals and hyperoxia exposure protocol}

Time-dated, pregnant Wistar rats (200-220 g) were purchased from the Center for Experimental Animals of China Medical University. All animal procedures were reviewed and approved by the Laboratory Animal Ethics Committee of China Medical University. Pups were delivered naturally at term gestation (22 days). The full-term newborn rats from twelve litters were randomly marked and assigned to two groups and were exposed to hyperoxia (95\% oxygen; experimental group) or normoxia (21\%; control group) beginning on the day of birth. The inhaled oxygen concentration was measured and recorded continuously with an analyzer equipped with a strip-chart recorder (model 572; Servomex, Norwood, MA). Humidity was routinely $60 \%-$ $70 \%$. Nursing rat dams were switched every 24 hours between the hyperoxic and normoxic chambers, to avoid oxygen toxicity and provide equal nutrition to each litter. Chambers were open for $10 \mathrm{~min}$ per day for weighing and cage cleaning. Pups were sacrificed and lungs were harvested at the end of 1, 3, 5 and 7 days of exposure.

\section{Lung histology}

The lungs of a random sample from each group were inflated by intratracheal instillation of $4 \%$ formaldehyde solution at a hydrostatic pressure of $18 \mathrm{cmH}_{2} \mathrm{O}$. The lungs were then fixed in $4 \%$ paraformaldehyde for $24 \mathrm{~h}$. Lung tissue was dehydrated with graded alcohol, placed in xylene for $1 \mathrm{~h}$ and then embedded in paraffin at $60^{\circ} \mathrm{C}$. Sections of lung $(5 \mu \mathrm{m})$ were stained with hematoxylin and eosin. Lung sections from all lungs were examined for any histological changes. From each section, 20 random areas were examined at $\times 400$ magnification. Within each field, lung injury was scored according to a new histologic acute lung injury (ALI) scoring system [29]. Histopathological evaluation was performed by an independent pathologist who was blinded to the experimental group. 


\section{Wet/dry lung weight ratio and excess lung water measurement}

The right upper lung lobes from random samples of two groups were excised at the end of the experiment and were weighed to determine the wet lung weight. After that, the tissues were placed in an oven and maintained at a temperature of $80^{\circ} \mathrm{C}$ for 48 hours before being weighed again to give final dry weight. The presence of pulmonary edema was examined by calculating the pulmonary wet/dry ratio (W/D).

To determine the extravascular water in the lung, a blood sample was obtained from cardiac puncture to measure the hemoglobin concentration. After the determination of lung wet weight, the tissue was homogenized and dried. Measurements of the hemoglobin concentration in the lung homogenate also allowed for the calculation of excess lung water according to the standard methods used as previously described [30,31].

\section{Measurement of bronchoalveolar epithelial permeability}

Pups were injected via the saphenous vein with fluorescein isothiocyanate-conjugated dextran 4000 (FD4) solution in PBS $(25 \mathrm{mg} / \mathrm{ml} ; 10 \mathrm{mg} / \mathrm{kg})$ after the anesthesia. Fifteen minutes later, the trachea was exposed, and the lungs were lavaged three times with $1 \mathrm{ml}$ of sterile saline per wash. The aliquots of bronchoalveolar lavage fluid (BALF) were collected. Blood was obtained by cardiac puncture, and the serum was collected by centrifugation at 3,000 rpm for $5 \mathrm{~min}$. The concentrations of FD4 in the BALF and serum (20 fold diluted by PBS) were determined with a spectrofluorometer (F-4500; Hitachi, Tokyo, Japan) using an excitation filter of $492 \mathrm{~nm}$ and an emission wavelength of $515 \mathrm{~nm}$. The BALF:serum fluorescence ratio was calculated and used as a measure of pulmonary epithelial permeability.

\section{Transmission electron microscopy study}

After dissection of lung, the specimens were fixed in $2.5 \%$ glutaraldehyde overnight at $4^{\circ} \mathrm{C}$. The tissues were washed in phosphate buffer ( $\mathrm{pH} 7.4)$, postfixed in $1 \%$ osmium tetroxide in phosphate buffer ( $\mathrm{pH} 7.4$ ), dehydrated in graded ethanol solutions, treated in propylene oxide and embedded in epoxy-resin embedding media. 60-nm thin transverse random sections were collected on single copper slot grids coated with parlodion, stained with uranyl acetate and lead citrate, and observed with a JEM1200EX transmission electron microscope (Hitachi Electronic Company, Japan). Images were documented by using Kodak SO163 EM film.

\section{Immunofluorescence staining}

Lungs of right middle lobe were inflated with $4 \%$ paraformaldehyde, soaked in 3\% paraformaldehyde for $3 \mathrm{~h}$ at $4^{\circ} \mathrm{C}$, cryoprotected in $30 \%$ sucrose for $12 \mathrm{~h}$ at $4^{\circ} \mathrm{C}$ and frozen at $80^{\circ} \mathrm{C}$. Frozen sections $(10 \mu \mathrm{m})$ were air dried and then washed three times with PBS. The sections were incubated with $0.5 \%$ Triton X-100 for $5 \mathrm{~min}$ at room temperature and then washed three times with PBS. The sections were then blocked with $10 \%$ goat serum for $30 \mathrm{~min}$ at $37^{\circ} \mathrm{C}$ and incubated with primary antibodies as follows: 1:200 diluted occludin (mouse monoclone antibody; Zymed Laboratories, South San Francisco, Calif) and 1:80 diluted ZO-1 (rabbit polyclone antibody; Zymed Laboratories, South San Francisco, Calif) together overnight at $4^{\circ} \mathrm{C}$. As negative controls, some sections were incubated in the absence of the primary antibodies. Tissue sections were washed four times with PBS-Triton. The second antibody against mouse was conjugated with Alexa fluor-488 (green fluorescence) and the anti-rabbit antibody with Alexa fluor-594 (red fluorescence) for $60 \mathrm{~min}$ at $37^{\circ} \mathrm{C}$. Sections were washed three times with PBS, and the nuclei were stained with DAPI (1:2000; Sigma Chemical) for $2 \mathrm{~min}$. After sufficient washes, images were captured by confocal laser scanning microscopy (MTC-600, BIO-RAD, USA).

\section{Immunoblotting}

Briefly, equal amounts of protein extract were mixed in $1 \times$ Laemmli buffer and boiled for $3 \mathrm{~min}$. The proteins were electrophoresed on $7.5 \%$ precast SDS-polyacrylamide gels (80 V for $120 \mathrm{~min}$ ). Then the proteins were transferred electrophoretically onto polyvinylidene difluoride (PVDF) membranes (40 V for $180 \mathrm{~min}$ ). The membranes were incubated for $1 \mathrm{~h}$ in 1:10 normal donkey serum to block nonspecific binding. The PVDF membranes were then incubated overnight at $4^{\circ} \mathrm{C}$ with primary antibodies occludin (1:500), ZO-1 (1:250), and $\beta$-actin $(1: 4,000)$ diluted in PBS-0.02\% Tween 20. Some samples were incubated in the solution without primary antibodies to be negative controls. After washing three times in PBS, the membranes were incubated at room temperature for $90 \mathrm{~min}$ with horseradish peroxidase-conjugated secondary antibody. The membrane was then washesd in PBS and impregnated with the enhanced chemiluminescence substrate (ECL kit; Santa Cruz Biotechnology) and was used to expose the radiograhic film. All the bands were scanned with Chemilmager 5500 V2.03 software, and IDVs were calculated by computerized image analysis system (Fluor Chen 2.0) and normalized to that of $\beta$-actin.

\section{RT-PCR}

Differential expression of occludin and ZO-1 was validated by a separate comparison of new subjects using RT-PCR. These studies compared spontaneously pups in normoxia group with pups exposured to hyperoxia. Briefly, lung tissue was dissected and homogenized. RNA was extracted (Invitrogen, Camarillo, Calif, USA) following the manufacturer's instructions. cDNA was generated from $1 \mu \mathrm{g}$ total of each sample (SuperScript III; Invitrogen). The sequences of following primers were designed 
by Primer Premier 5.0 software (Premier Biosoft International, Palo Alto, Calif) and are shown in Table 1. The amplification reaction was carried out in 30 cycles at $95^{\circ}$ $\mathrm{C}$ for $45 \mathrm{~s}, 55^{\circ} \mathrm{C}$ for $45 \mathrm{~s}$, and $75^{\circ} \mathrm{C}$ for $1 \mathrm{~min}$ in a thermal cycler. PCR products were electrophoresed on $2.5 \%$ agarose gels. Gels were photographed by a Chemilmager 5500 gel image analysis instrument (Alpha Innotech, USA). The integrated density values (IDVs) of PCR product bands were calculated by computerized image analysis system (Fluor Chen 2.0) and expressed relative to that of $\beta$-actin.

\section{Statistical analysis}

SPSS 18.0 software was used for this statistical analysis. Data were summarized as mean $\pm \mathrm{SD}$. Student $t$-test was used to determine the significant difference between two groups. One-way analysis of variance (ANOVA) and post hoc comparisons (Bonferroni test) were used to determine the significant difference among multiple groups. A $P$ value of less than 0.05 was considered to be statistically significant.

\section{Results}

Lung histology shows levels of lung injury after different days of hyperoxia exposure

Formalin-fixed paraffin-embedded lung tissues from newborn rats after different days of hyperoxia or normoxia exposure were treated with hematoxylin-eosin (HE) staining. On the first day, the alveolar-like structures in full-term newborn rat lungs exposed to normoxia were irregular, and a small number of septa could be seen (Figure 1A). There was no obvious difference in pulmonary morphology in newborn rats exposed to hyperoxia compared with that of the normoxia group on day 1 (Figure 1B). The septa became thinner and the numbers increased in the normoxia group on the third day (Figure 1C). The septa were thickened and vascular congestion was also observed. There was neutrophil infiltration in the interstitial space in the hyperoxia group on day 3 (Figure 1D). On the fifth day, the alveoli grew in number, and the septa continued to be thinner in the normoxia group (Figure 1E). After 5 days of hyperoxia, histological evaluation of lung tissue in newborn rats revealed increased neutrophil infiltration in the interstitium. There was more severe interstitial edema and a few inflammatory cells infiltrating the alveolar space (Figure 1F). The alveoli were regular and uniformly distributed in newborn rats exposed to normoxia on the seventh day (Figure 1G). In the hyperoxia group, there was increased loss of pulmonary epithelial architecture, more inflammatory cells and red blood cell infiltration in the alveolar space with proteinaceous debris filling the airspaces on day 7 (Figure 1H). After 7 days of hyperoxia, the lung injury appeared more severe than that observed on day 3 or day 5 . Treatment with hyperoxia for 3 days resulted in a less severe injury to lung tissue compared with that observed on day 5. The observation demonstrated that more severe lung injury happened in newborn rats with longer hyperoxia exposure. Lung injury scores, shown in Figure 1I, supported the observation that the lung injury level of newborn rats treated with hyperoxia was time-dependent.

\section{Effect of hyperoxia exposure on pulmonary edema}

Pulmonary edema formation, indicative of injury to the pulmonary epithelial barrier, often happens with an increase in the total water W/D ratio. Our study revealed there was no difference in W/D ratio between the normoxia group and the hyperoxia group on the first day $(P$ $>0.05)$. On the third day, W/D ratio in the hyperoxia groups was significantly higher than that of the normoxia group $(P<0.05)$. After 5 days of hyperoxia exposure, W/ $D$ ratio was much higher than that of the normoxia group $(P<0.01)$. There was significant increase in $\mathrm{W} / \mathrm{D}$ ratio in newborn rats with 7 days of hyperoxia exposure compared with that in corresponding normoxia group $(P$ $<0.01$ ) (Figure 2A).

The measurement of extravascular lung water content is also valuable in studies of mechanisms of increased permeability pulmonary edema. The extravascular lung water content in newborn rats with hyperoxia exposure was higher than that with normoxia exposure and the results were almost the same with the lung W/D ratio (Figure 2B).

\section{Hyperoxia exposure decreases pulmonary epithelial barrier function}

Generally, pulmonary epithelial barrier function decreases with an increase of bronchoalveolar epithelial permeability. We monitored the changes in pulmonary epithelial barrier function by examining the effect of hyperoxia exposure on bronchoalveolar epithelial permeability. Bronchoalveolar epithelial permeability was determined by measuring the leakage of FD4 from serum into BALF in rats (Figure 3). On the first day, there was no obvious difference in the

Table 1 List of primers used for measuring gene expression by RT-PCR

\begin{tabular}{lcc}
\hline Gene name & Forward Primer & Reverse Primer \\
\hline Occludin & TTGGGAGCCTTGACATCTTGTTC & GCCATACATGTCATTGCTTGGTG \\
ZO-1 & CCATCTTGGACCGATTGCTG & TAATGCCCGAGCTCCGATG \\
$\beta$-actin & GGAGATTACTGCCCTGGCTCCTA & GACTCATCGTACTCCTGCTTGCTG \\
\hline
\end{tabular}




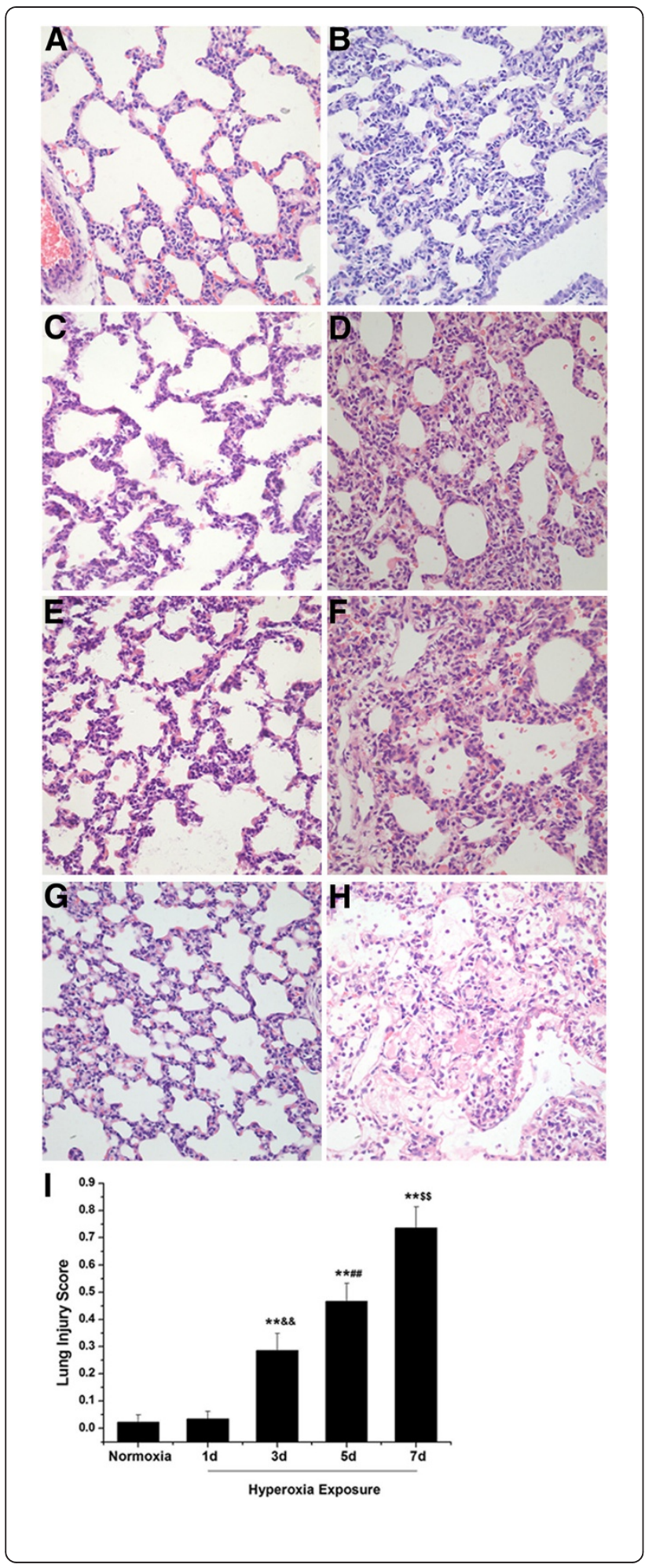

BALF:serum fluorescence ratios between the hyperoxia and normoxia groups $(P>0.05)$. After 3 days of hyperoxia exposure, the ratios of BALF:serum fluorescence in newborn rats were significantly higher than those in normoxia group $(P<0.05)$. The BALF:serum fluorescence ratios in

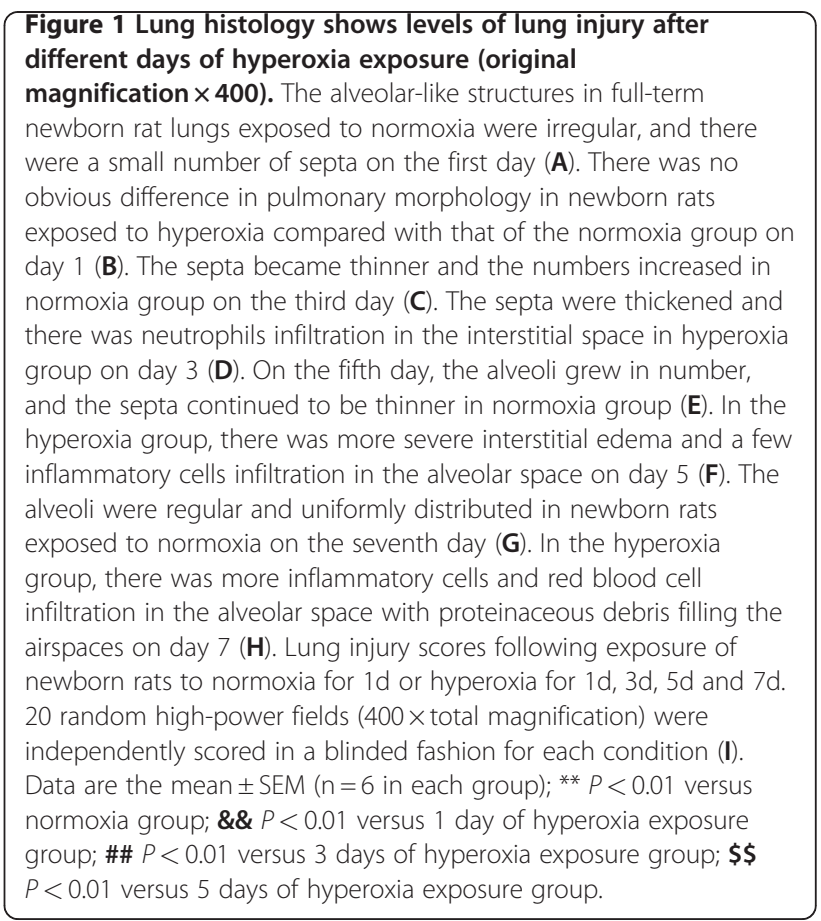

hyperoxia group were approximately 2 -fold higher than those in the control group on day $5(P<0.01)$. On the seventh days, the BALF:serum FD4 ratios in hyperoxia group further increased and were more than 3-fold higher than those in the normoxia group $(P<0.01)$. Prolonged treatment with hyperoxia exposure significantly increased the bronchoalveolar epithelial permeability.

\section{Morphological alterations of tight junction in alveolar epithelia during hyperoxia exposure}

Since hyperoxia exposure increased the bronchoalveolar epithelial permeability in newborn rats, it might alter the morphology of tight junction in the alveolar epithelium. The integrity of pulmonary epithelium and morphological alterations of tight junction were studied by transmission electron microscopy in newborn rats with normoxia and hyperoxia exposure. Random sampling of left lung from newborn rats exposed to normoxia showed obvious and frequent junctions between all epithelial cells which were extremely tight (Figure 4A). The tight junctions were ultrastructurally normal (Figure 4B). In contrast, after treatment with hyperoxia for 7 days, random sampling of left lungs from newborn rats showed loss of tight junctions between alveolar epithelial cells (Figure 4C). The tight junctions were irregularly widened (Figure 4D). Epithelial cells appeared retracted from each other with clear paracellular gaps. After 5 days of hyperoxia exposure, the tight junctions were also widened but to a much less extent (Figure 4E, F). Morphology of tight junction showed no observable changes in newborn rats exposed to hyperoxia for 1 day or 3 day (Figure 4G, H). 

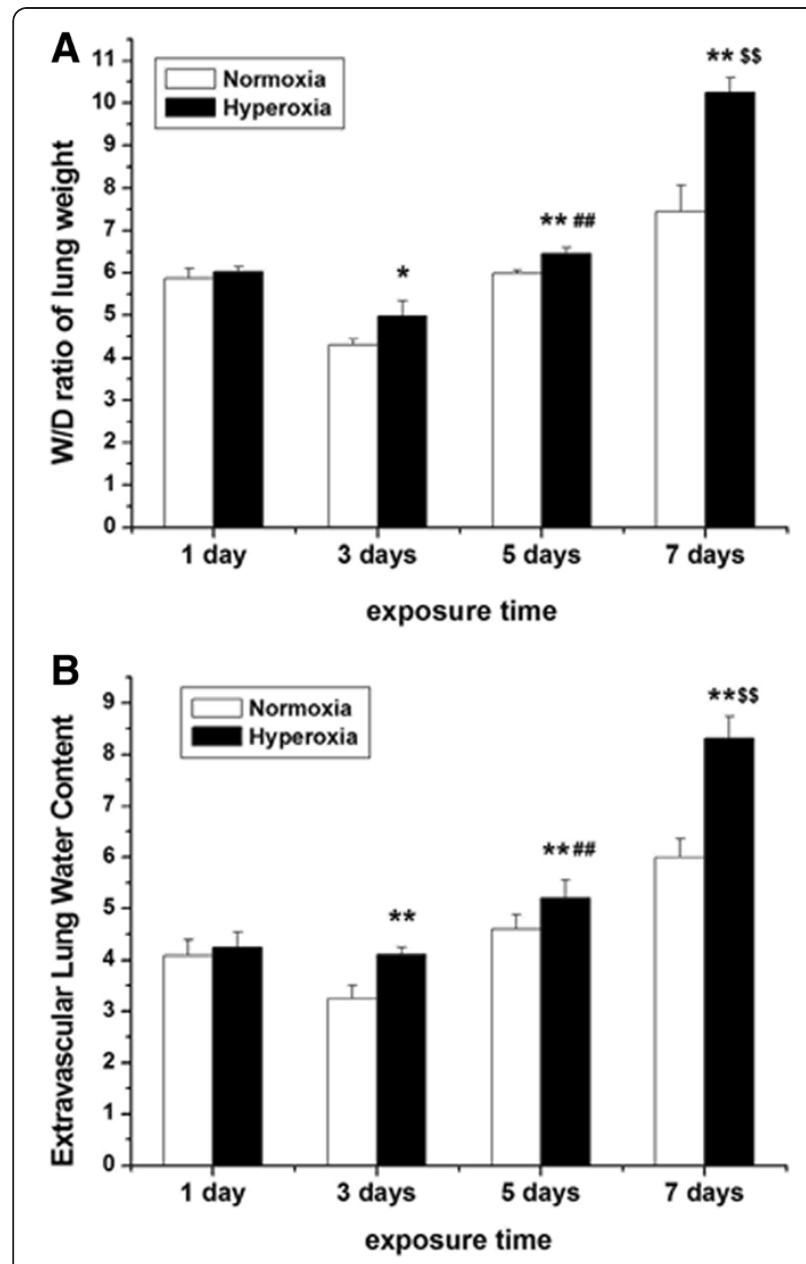

Figure 2 Effect of hyperoxia exposure on pulmonary edema after different days of hyperoxia exposure. Effects of hyperoxia exposure on pulmonary edema were determined by the wet-to-dry (W/D) ratio of lung weight $(\mathbf{A})$ and extravascular lung water content (B) following exposure of newborn rats to normoxia or hyperoxia for 1, 3, 5 and 7 days. Hyperoxia significantly increased the W/D ratio of lung weight and extravascular lung water content compared with normoxia on 5 and 7 days. Data are the mean \pm SEM $(n=6$ in each group); ${ }^{*} P<0.05$, ${ }^{* *} P<0.01$ versus normoxia group; \#\# $P<0.01$ versus 3 days of hyperoxia exposure group; $\$ \$ P<0.01$ versus 5 days of hyperoxia exposure group.

Effects of hyperoxia exposure on the localization and expression of ZO-1 and occludin in alveolar epithelia The morphology of tight junctions in the alveolar epithelium altered when exposed to hyperoxia. Then the localization and expression of tight junction proteins in the alveolar epithelium might change. ZO-1 and occludin, which are two of the best-characterized tight junction proteins, were examined by immunofluorescence staining from frozen sections of the right middle lobe. ZO-1 was localized in lung tissue from rats with normoxia exposure as a continuous line along the boundaries between neighboring bronchial and alveolar epithelial cells (Figure 5A). Within one day of hyperoxia exposure, ZO-1 distribution in tight junctions was not obviously diminished. After 3 and 5 days, hyperoxia exposure induced partial breakdown of membrane staining and decreased cytoplasm staining of ZO-1 in the alveolar epithelium (Figure 5B). Treatment with hyperoxia for 7 days induced a fragmented staining pattern on the membrane (Figure $5 \mathrm{C}$ ). The features of occludin immunostaining were similar to that of ZO-1. A consecutive line along the membrane and diffuse in the cytoplasm staining of occludin was shown in the alveolar epithelium of control rats (Figure 5D). The intensity of this staining was dramatically reduced in lung tissues from rats which were exposed to hyperoxia for 7 days (Figure $5 \mathrm{E}$ ).

Furthermore, we detected the protein expression of ZO-1 and occludin from lung tissue by immunoblotting. Hyperoxia exposure can induce different levels of tight junction proteins expression in neonatal rat lung tissue after $1-7$ days of duration. The protein expression levels of ZO-1 increased slightly after one day of hyperoxia exposure and clear ZO-1 bands were apparent in both experimental and control group on day 1 . However, the decrease of ZO-1 expression was statistically significant after 3 days of hyperoxia exposure $(P<0.01)$. Further decreased expression of these proteins was observed in newborn rats with prolonged hyperoxia exposure (Figure 5F, G). The decrease of occludin expression was statistically significant on day 5 $(P<0.05)$. After 7 days of hyperoxia exposure, the expression dramatically declined and was virtually undetectable by Western blotting (Figure 5H, I).

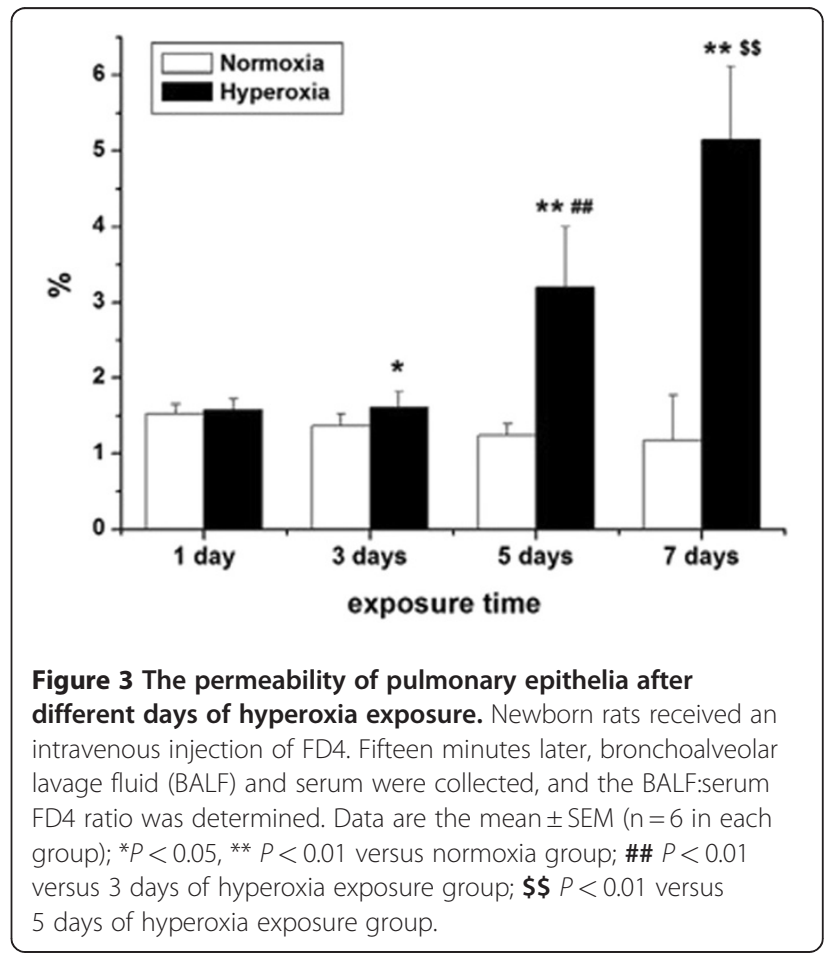




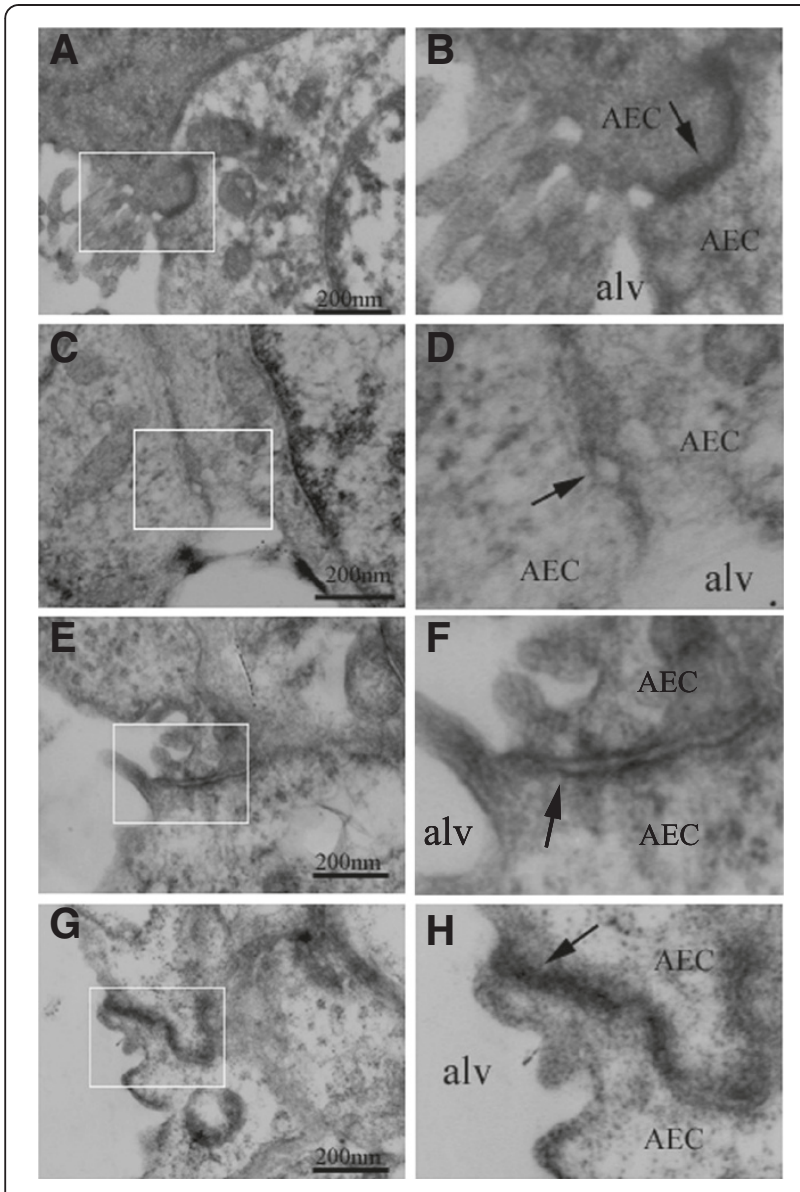

Figure 4 Hyperoxia exposure disrupts alveolar epithelial tight junctions. The integrity of pulmonary epithelium and morphological alterations of tight junction were studied by transmission electron microscopy in both normoxia and hyperoxia groups. Tight junctions between pulmonary epithelial cells were extremely tight in the lungs from normoxia-treated newborn rats (A and $\mathbf{B})$. After treatment with hyperoxia for $\mathbf{7}$ days, tight junctions were irregularly widened $(\mathbf{C}$ and D). Tight junctions were open between epithelial cells in newborn rats with hyperoxia for 5 days, and paracellular gaps were visible between alveolar epithelial cells (E and $\mathbf{F}$ ). Intact tight junctions remained between alveolar epithelial cells in newborn rats exposed to hyperoxia for 1 and 3 days $(\mathbf{G}$ and $\mathbf{H}$ ).

\section{Hyperoxia exposure decreases the mRNA expression of} ZO-1 and occludin in the lung of newborn rats

The messenger RNA expressions of ZO-1 and occludin were further examined in lung tissue by RT-PCR. The results showed that 1 day of hyperoxia exposure significantly depressed the messenger RNA levels of ZO-1 $(P<0.01)$. The messenger RNA levels were markedly downregulated in newborn rats with prolonged hyperoxia exposure $(P<0.01)$ (Figure 6A, B). The decrease of occludin mRNA level was statistically significant after 3 or 5 days of hyperoxia exposure $(P<0.05)$. Treatment with hyperoxia for 7 days had the greatest depressive effect on these messenger RNA levels $(P<0.01)$ (Figure $6 \mathrm{C}, \mathrm{D})$. The results were consistent with the changes of these protein expressions in lung tissue.

\section{Disscussion}

Although hyperoxia exposure is the most common cause of lung injury for neonates requiring respiratory support, the molecular and cellular mechanisms underlying this phenomenon remain elusive. Non-cardiogenic pulmonary edema and diffuse infiltration of inflammatory cells have been confirmed as the major pathologic findings in hyperoxia-induced acute lung injury $[9,10]$. Previous studies on mechanisms related to these pathologic changes are mostly focused on abnormal secretion of cytokines, and imbalance between oxidation and antioxidation $[2,8,32,33]$. However, treatment against those factors is insufficient [34-36]. Furthermore, the molecular mechanisms involved in disruption of the pulmonary epithelial barrier itself are seldom investigated in neonatal hyperoxic lung injury and no such research is concerned with how tight junctions change during the disease. Thus, our study was undertaken to explore possible mechanisms of impairment of pulmonary epithelial integrity and find what role tight junctions play in newborn animals with hyperoxia exposure.

Our study, for the first time, demonstrated alterations in the structure and function of tight junctions in hyperoxiainduced lung injury in newborn animals. We observed that hyperoxia exposure impaired pulmonary epithelial tight junctions with increased pulmonary epithelial permeability in an in vivo model. Also, we found that the expression of occludin and ZO-1 decreased during hyperoxia exposure and the alterations were consistent with changes in the barrier function of the bronchoalveolar epithelium, suggesting these key tight junction proteins might play important roles in the disruption of epithelial integrity in hyperoxic lung injury in newborn rats.

This study provided new evidence that hyperoxia exposure led to inflammation and pulmonary edema. According to the histologic findings, we confirmed that proteinaceous fluid and neutrophil infiltration in lung tissue markedly increased after hyperoxia exposure. Pulmonary neutrophil sequestration and transmigration are demonstrated as typical manifestations of acute lung injury $[37,38]$. In this study, the results of lung W/D ratio and extravascular lung water content reached a common consensus that, there was obvious pulmonary edema in newborn rats with prolonged hyperoxia exposure, which were comparable with the severity of changes in pathology. These results are in accordance with previous studies which show polymorphonuclear cell infiltration and edema formation in hyperoxia-induced ALI $[39,40]$.

We also showed that hyperoxia exposure in newborn rats significantly increased bronchoalveolar epithelial permeability (about 2-4 fold). The magnitude of the 

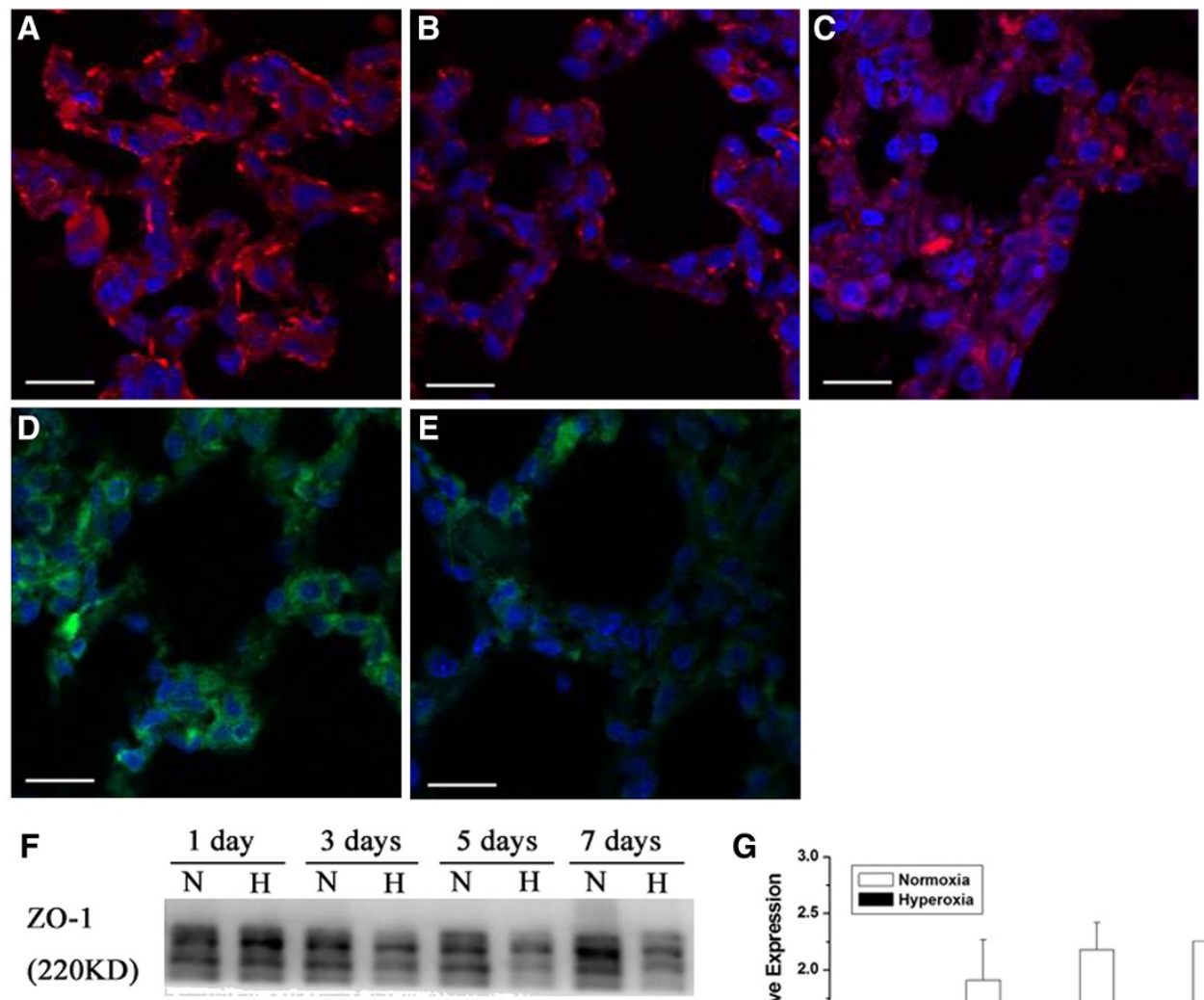

B-actin

(43KD)
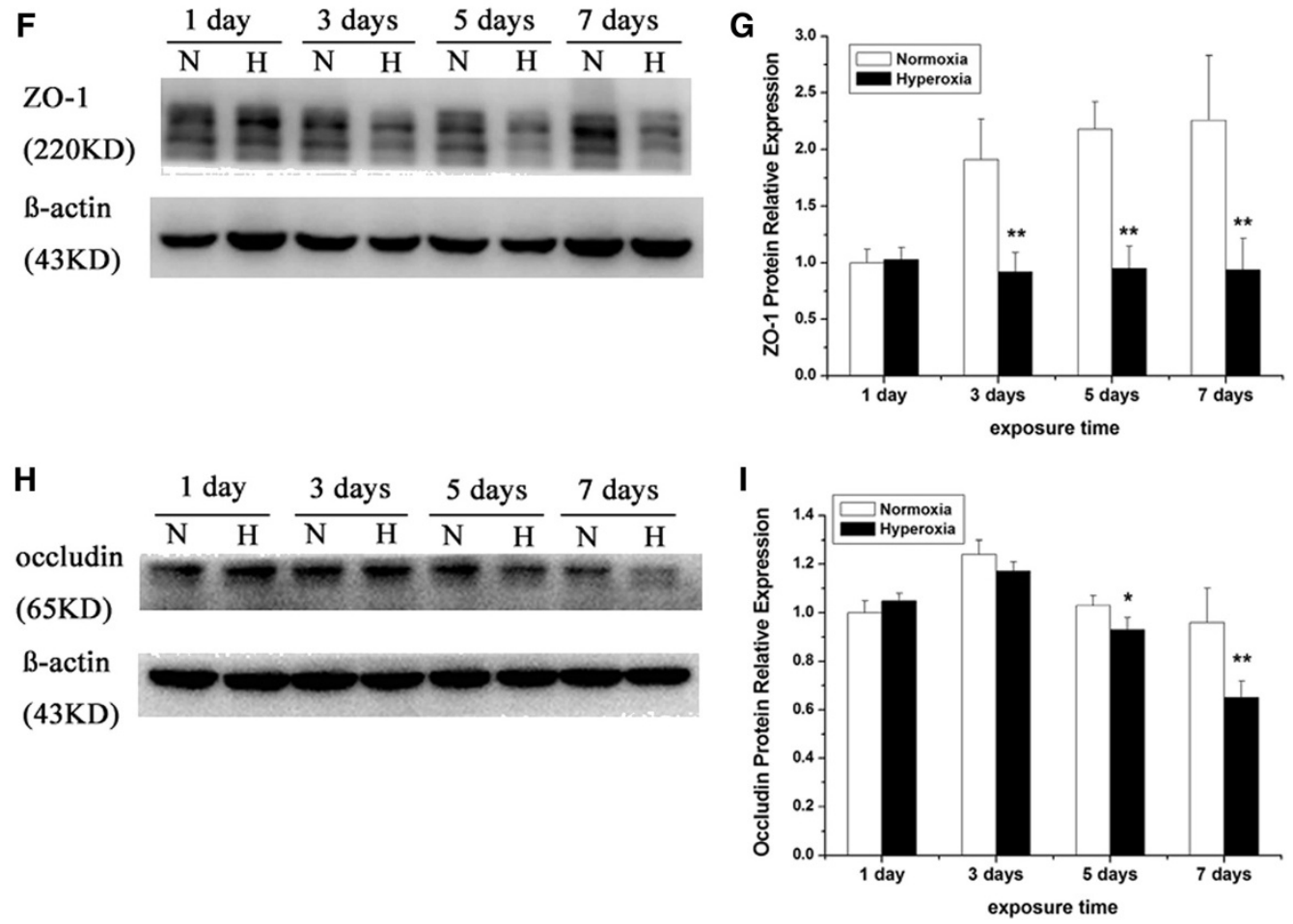

Figure 5 Hyperoxia exposure alters location and expression of ZO-1 and occludin in alveolar epithelial. Immunostaining and western blot analysis were performed to identify alteration in location and expression of ZO-1 and occludin in alveolar epithelial. A continuous line along the membrane and diffuse in the cytoplasm staining of ZO-1 and occludin was shown in the alveolar epithelium of control rats (A, D). After 3 and 5 days, hyperoxia exposure induced partial breakdown of membrane staining and decreased cytoplasm staining of ZO-1 in the alveolar epithelium (B). The staining intensity of ZO-1 and occludin was dramatically reduced in lung tissues from rats which were exposed to hyperoxia for 7 days (C, E). Bars represent $50 \mu \mathrm{m}$. After 7 days of hyperoxia exposure, the expression of ZO-1 and occludin dramatically declined and was virtually undetectable examined by Western blotting. $\beta$-actin immunoreactive bands were used to demonstrate equal loading $(F, H)$. Densitometric quantification of ZO-1 and occludin protein levels was expressed as fold increase compared with normoxia for 1 day group (set as 1) $(G, I)$. Values are presented as means $\pm S D ;{ }^{*} P<0.05$, ${ }^{* *} P<0.01$ versus normoxia group. The results shown are representative of experiments that were repeated 4 times. 


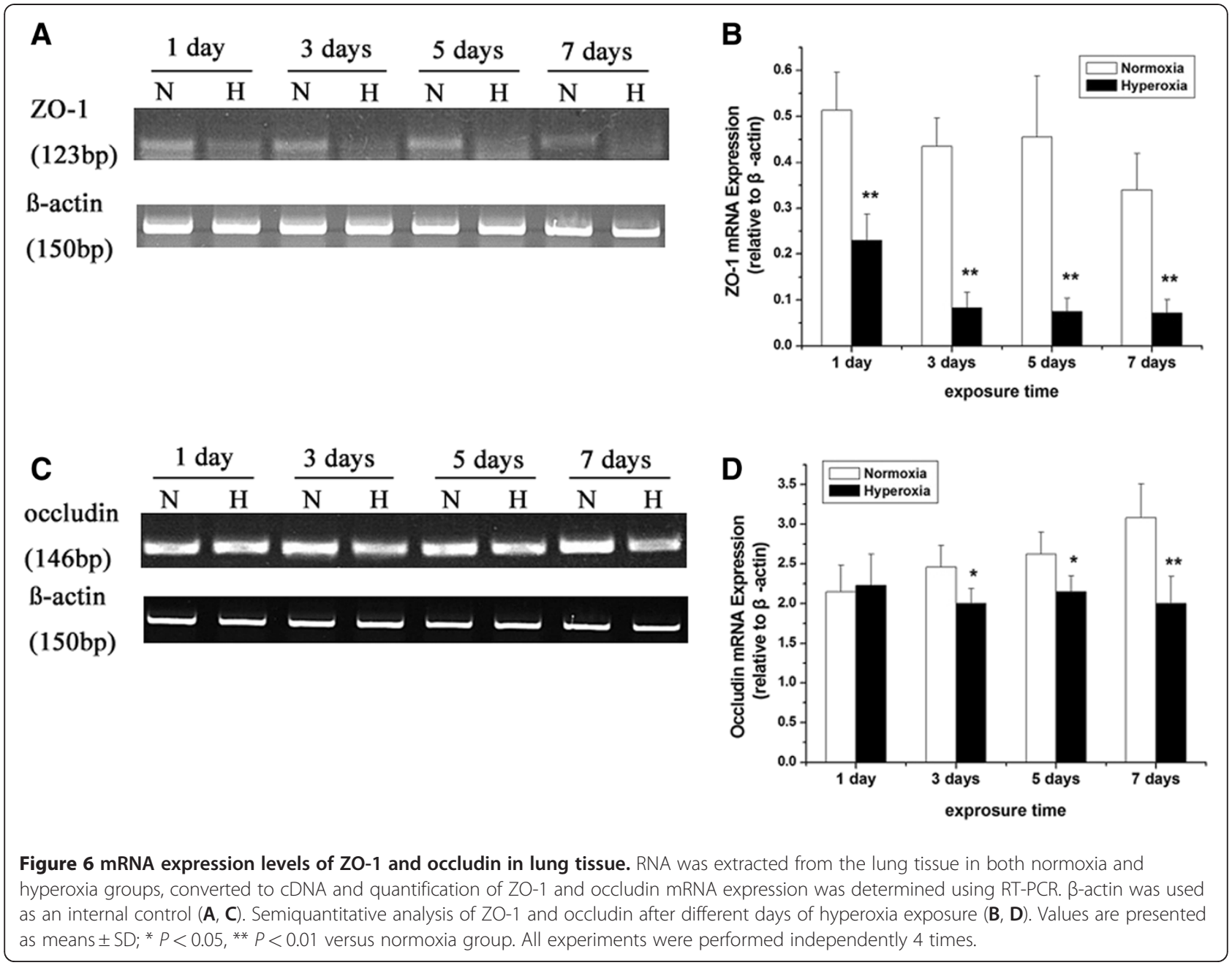

functional alteration in pulmonary epithelial barrier peaked after 7 days of hyperoxia exposure while the BALF:serum FD4 ratio was almost unchanged on day 1 . The data demonstrated treatment with hyperoxia for prolonged duration contributed to more serious lung injury. Our findings are consitent with other studies which show hyperoxia increases pulmonary permeability $[41,42]$. It has been determined that severe lung injury and inflammatory reaction was induced by $95 \% \mathrm{O} 2$ for 3 and 7 days with protein leak across the alveolar epithelium increasing in rat pups [43]. BALF albumin levels and neutrophil count are significantly increased in adult rats with hyperoxia exposure, suggesting the alteration in alveolar epithelial barrier function [44].

Since maintenance of normal pulmonary barrier function depends on intact epithelial tight junctions [24,26], transmission electron microscopy was used in this study to examine their morphological alterations. We observed that treatment with hyperoxia in newborn rats destroyed the structure of tight junctions. Using immunofluorescence, we showed that hyperoxia exposure markedly disrupted the membrane localization and downregulated the cytoplasm expression of the key tight junction proteins occludin and ZO-1 in the alveolar epithelium and the results were confirmed by immunoblotting and RT-PCR. Functional opening of tight junction barrier and downregulation of occludin and ZO-1 protein expression have been also found in acute lung injury in adult animals $[27,28]$. Previous studies in intestinal epithelia have shown that morphology of tight junctions change through downregulation and localization shift of occluding [24,25].

Furthermore, our study showed that prolonged hyperoxia exposure enhanced the structure destruction of epithelial tight junctions while decreasing in occludin and ZO-1 expression was more obvious. This temporal pattern of changes in the expression of key tight junction proteins was paralleled by changes in the barrier function of the bronchoalveolar epithelium, which suggested decreased synthesis of occludin and ZO-1 at a transcriptional level may be a mechanism for the rapid disruption of epithelial integrity in newborn animals with hyperoxia-induced lung injury. However, abnormal structure of the tight junction 
was not found in groups with 1 or 3 days of hyperoxia exposure. Also, pathohistologic findings showed there was no inflammation and protein leakage until the third day of hyperoxia exposure. This phenomenon suggests that mildto-moderate deterioration of these proteins may not be enough to change the morphology of tight junctions and cause severe lung edema.

A preliminary study has given some clue that oxidative stress induces tyrosine phosphorylation and dissociates the occludin-ZO-1 complexes from the cytoskeleton by a tyrosine kinase-dependent mechanism [45]. Whether hyperoxia disrupts alveolar epithelial barrier function by means of tyrosine phosphorylation and dissociation of occludin-ZO-1 complexes needs further study.

\section{Conclusion}

Taken together, this study demonstrates that hyperoxia exposure in newborn rats impairs the structure and function of alveolar epithelial tight junctions and the changes are enhanced with prolonged hyperoxia exposure. Our data demonstrate occludin and ZO-1 expressions decrease during hyperoxia-induced acute lung injury in neonatal animals and the alterations are consistent with the severity of lung injury. Therefore, we propose that the disruption of the pulmonary epithelial barrier induced by hyperoxia is, at least in part, due to massive deterioration in the expression and localization of key TJ proteins. How deterioration of these proteins impairs the function of pulmonary epithelial barrier needs further study.

\section{Abbreviations \\ BALF: Bronchoalveolar lavage fluid; BPD: Bronchopulmonary dysplasia; FD4: Fluorescein isothiocyanate-conjugated dextran 4000; HALI: Hyperoxic acute lung injury; TJ: Tight junction; ZO-1: Zonula occludens 1.}

\section{Competing interests}

The authors declare that they have no competing interests.

\begin{abstract}
Authors' contributions
KY performed all the experiments, analyzed the data, and wrote the manuscript. JF and XXue participated in the design of the experiments and manuscript revision. $X X u$ and SX participated in the animal studies and coordinated the immunostaining studies. $Z Y$ and $X Y$ helped to perform the statistical analysis. All authors read and approved the final manuscript.
\end{abstract}

\section{Acknowledgements}

This work was supported by grant from the Natural Science Foundation of China (No: 30872781).

\section{Author details \\ 'Department of Pediatrics, Shengjing Hospital of China Medical University, Shenyang 110004, China. ${ }^{2}$ Department of Urology, Shengjing Hospital of China Medical University, Shenyang 110004, China. ${ }^{3}$ Department of Pediatrics, Fengtian Hospital Affiliated to Shenyang Medical College, Shenyang 110024, China.}

Received: 3 February 2012 Accepted: 4 May 2012

Published: 4 May 2012

\section{References}

1. Jobe AH, Bancalari E: Bronchopulmonary dysplasia. Am J Respir Crit Care Med 2001, 163:1723-1729.
2. Bancalari $E$, Claure N, Sosenko IR: Bronchopulmonary dysplasia: changes in pathogenesis, epidemiology and definition. Semin Neonatol 2003, 8:63-71.

3. Smith VC, Zupancic JA, McCormick MC, Croen LA, Greene J, Escobar GJ, Richardson DK: Rehospitalization in the first year of life among infants with bronchopulmonary dysplasia. J Pediatr 2004, 144:799-803.

4. Doyle LW: Respiratory function at age 8-9 years in extremely low birthweight/very preterm children born in Victoria in 1991-1992. Pediatr Pulmonol 2006, 41:570-576.

5. Doyle LW, Faber B, Callanan C, Freezer N, Ford GW, Davis NM: Bronchopulmonary dysplasia in very low birth weight subjects and lung function in late adolescence. Pediatrics 2006, 118:108-113.

6. Short EJ, Klein NK, Lewis BA, Fulton S, Eisengart S, Kercsmar C, Baley J, Singer $\mathrm{LT}$ : Cognitive and academic consequences of bronchopulmonary dysplasia and very low birth weight: 8-year-old outcomes. Pediatrics 2003, 112:e359.

7. Anderson PJ, Doyle LW: Neurodevelopmental outcome of bronchopulmonary dysplasia. Semin Perinatol 2006, 30:227-232.

8. Kotecha S, Chan B, Azam N, Silverman M, Shaw RJ: Increase in interleukin-8 and soluble intercellular adhesion molecule-1 in bronchoalveolar lavage fluid from premature infants who develop chronic lung disease. Arch Dis Child Fetal Neonatal Ed 1995, 72:F90-F96.

9. Coalson JJ: Pathology of new bronchopulmonary dysplasia. Semin Neonatol 2003, 8:73-81

10. Warner BB, Stuart LA, Papes RA, Wispe JR: Functional and pathological effects of prolonged hyperoxia in neonatal mice. Am J Physiol 1998, 275: L110-L117.

11. Corne J, Chupp G, Lee CG, Homer RJ, Zhu Z, Chen Q, Ma B, Du Y, Roux F, McArdle J, et al: IL-13 stimulates vascular endothelial cell growth factor and protects against hyperoxic acute lung injury. J Clin Invest 2000, 106:783-791.

12. Altemeier WA, Sinclair SE: Hyperoxia in the intensive care unit: why more is not always better. Curr Opin Crit Care 2007, 13:73-78.

13. Crandall ED, Matthay MA: Alveolar epithelial transport. Basic science to clinical medicine. Am J Respir Crit Care Med 2001, 163:1021-1029.

14. Ware LB, Matthay MA: Alveolar fluid clearance is impaired in the majority of patients with acute lung injury and the acute respiratory distress syndrome. Am J Respir Crit Care Med 2001, 163:1376-1383.

15. Schneeberger EE, Lynch RD: Structure, function, and regulation of cellular tight junctions. Am J Physiol 1992, 262:L647-L661.

16. Gonzalez-Mariscal L, Betanzos A, Nava P, Jaramillo BE: Tight junction proteins. Prog Biophys Mol Biol 2003, 81:1-44.

17. McCarthy KM, Skare IB, Stankewich MC, Furuse M, Tsukita S, Rogers RA Lynch RD, Schneeberger EE: Occludin is a functional component of the tight junction. J Cell Sci 1996, 109(Pt 9):2287-2298.

18. Chen Y, Merzdorf C, Paul DL, Goodenough DA: $\mathrm{COOH}$ terminus of occludin is required for tight junction barrier function in early Xenopus embryos. J Cell Biol 1997, 138:891-899.

19. Denker BM, Nigam SK: Molecular structure and assembly of the tight junction. Am J Physiol 1998, 274:F1-F9.

20. Ohtake K, Maeno T, Ueda H, Ogihara M, Natsume H, Morimoto Y: Poly-Larginine enhances paracellular permeability via serine/threonine phosphorylation of ZO-1 and tyrosine dephosphorylation of occludin in rabbit nasal epithelium. Pharm Res 2003, 20:1838-1845.

21. Ye L, Martin TA, Parr C, Harrison GM, Mansel RE, Jiang WG: Biphasic effects of 17-beta-estradiol on expression of occludin and transendothelial resistance and paracellular permeability in human vascular endothelial cells. J Cell Physiol 2003, 196:362-369.

22. Chasiotis H, Wood CM, Kelly SP: Cortisol reduces paracellular permeability and increases occludin abundance in cultured trout gill epithelia. Mol Cell Endocrinol 2010, 323:232-238.

23. Raleigh DR, Boe DM, Yu D, Weber CR, Marchiando AM, Bradford EM, Wang Y, Wu L, Schneeberger EE, Shen L, Turner JR: Occludin S408 phosphorylation regulates tight junction protein interactions and barrier function. J Cell Biol 2011, 193:565-582.

24. Han X, Fink MP, Yang R, Delude RL: Increased iNOS activity is essential for intestinal epithelial tight junction dysfunction in endotoxemic mice. Shock 2004, 21:261-270.

25. Noth R, Lange-Grumfeld J, Stuber E, Kruse ML, Ellrichmann M, Hasler R, Hampe J, Bewig B, Rosenstiel P, Schreiber S, Arlt A: Increased intestinal permeability and tight junction disruption by altered expression and localization of occludin in a murine graft versus host disease model. BMC Gastroenterol 2011, 11:109. 
26. Gon Y, Wood MR, Kiosses WB, Jo E, Sanna MG, Chun J, Rosen H: S1P3 receptor-induced reorganization of epithelial tight junctions compromises lung barrier integrity and is potentiated by TNF. Proc Natl Acad Sci U S A 2005, 102:9270-9275.

27. Mazzon E, Cuzzocrea S: Role of TNF-alpha in lung tight junction alteration in mouse model of acute lung inflammation. Respir Res 2007, 8:75.

28. Zhang YL, Li QQ, Guo W, Huang Y, Yang J: Effects of chronic ethanol ingestion on tight junction proteins and barrier function of alveolar epithelium in the rat. Shock 2007, 28:245-252.

29. Matute-Bello G, Downey G, Moore BB, Groshong SD, Matthay MA, Slutsky AS, Kuebler WM: An official American Thoracic Society workshop report: features and measurements of experimental acute lung injury in animals. Am J Respir Cell Mol Biol 2011, 44:725-738.

30. Li T, Koshy S, Folkesson HG: Involvement of \{alpha\}ENaC and Nedd4-2 in the conversion from lung fluid secretion to fluid absorption at birth in the rat as assayed by RNA interference analysis. Am J Physiol Lung Cell Mol Physiol 2007, 293:L1069-L1078.

31. Song Y, Fukuda N, Bai C, Ma T, Matthay MA, Verkman AS: Role of aquaporins in alveolar fluid clearance in neonatal and adult lung, and in oedema formation following acute lung injury: studies in transgenic aquaporin null mice. J Physiol 2000, 525(Pt 3):771-779.

32. Ben-Ari J, Makhoul IR, Dorio RJ, Buckley S, Warburton D, Walker SM: Cytokine response during hyperoxia: sequential production of pulmonary tumor necrosis factor and interleukin-6 in neonatal rats. Isr Med Assoc J 2000, 2:365-369.

33. Auten RL, Davis JM: Oxygen toxicity and reactive oxygen species: the devil is in the details. Pediatr Res 2009, 66:121-127.

34. Doyle LW, Ehrenkranz RA, Halliday HL: Dexamethasone treatment in the first week of life for preventing bronchopulmonary dysplasia in preterm infants: a systematic review. Neonatology 2010, 98:217-224.

35. Dani C, Cecchi A, Bertini G: Role of oxidative stress as physiopathologic factor in the preterm infant. Minerva Pediatr 2004, 56:381-394.

36. Comhair SA, Erzurum SC: Antioxidant responses to oxidant-mediated lung diseases. Am J Physiol Lung Cell Mol Physiol 2002, 283:L246-L255.

37. Deng H, Mason SN, Auten RL Jr: Lung inflammation in hyperoxia can be prevented by antichemokine treatment in newborn rats. Am J Respir Crit Care Med 2000, 162:2316-2323.

38. Reutershan J, Basit A, Galkina EV, Ley K: Sequential recruitment of neutrophils into lung and bronchoalveolar lavage fluid in LPS-induced acute lung injury. Am J Physiol Lung Cell Mol Physiol 2005, 289:L807-L815.

39. Jankov RP, Johnstone L, Luo X, Robinson BH, Tanswell AK: Macrophages as a major source of oxygen radicals in the hyperoxic newborn rat lung. Free Radic Biol Med 2003, 35:200-209.

40. Yi M, Jankov RP, Belcastro R, Humes D, Copland I, Shek S, Sweezey NB, Post M, Albertine KH, Auten RL, Tanswell AK: Opposing effects of $60 \%$ oxygen and neutrophil influx on alveologenesis in the neonatal rat. Am J Respir Crit Care Med 2004, 170:1188-1196.

41. Ahamed K, Epaud R, Holzenberger M, Bonora M, Flejou JF, Puard J, Clement A Henrion-Caude A: Deficiency in type 1 insulin-like growth factor receptor in mice protects against oxygen-induced lung injury. Respir Res 2005, 6:31.

42. Lozon TI, Eastman AJ, Matute-Bello G, Chen P, Hallstrand TS, Altemeier WA: PKR-dependent CHOP induction limits hyperoxia-induced lung injury. Am J Physiol Lung Cell Mol Physiol 2011, 300:L422-L429.

43. Wang M, Luo Z, Liu S, Li L, Deng X, Huang F, Shang L, Jian C, Yue S: Glutamate mediates hyperoxia-induced newborn rat lung injury through N-methyl-D-aspartate receptors. Am J Respir Cell Mol Biol 2009, 40:260-267.

44. Aggarwal NR, D'Alessio FR, Tsushima K, Files DC, Damarla M, Sidhaye VK, Fraig MM, Polotsky VY, King LS: Moderate oxygen augments lipopolysaccharide-induced lung injury in mice. Am J Physiol Lung Cell Mol Physiol 2010, 298:L371-L381.

45. Rao RK, Basuroy S, Rao VU, Karnaky KJ Jr, Gupta A: Tyrosine phosphorylation and dissociation of occludin-ZO-1 and E-cadherin-betacatenin complexes from the cytoskeleton by oxidative stress. Biochem $J$ 2002, 368:471-481.

doi:10.1186/1465-9921-13-36

Cite this article as: You et al:: Hyperoxia disrupts pulmonary epithelial barrier in newborn rats via the deterioration of occludin and ZO-1. Respiratory Research 2012 13:36.

\section{Submit your next manuscript to BioMed Central and take full advantage of:}

- Convenient online submission

- Thorough peer review

- No space constraints or color figure charges

- Immediate publication on acceptance

- Inclusion in PubMed, CAS, Scopus and Google Scholar

- Research which is freely available for redistribution

Submit your manuscript at www.biomedcentral.com/submit
( BioMed Central 